Short communication

\title{
Electrosprayed whey protein-based nanocapsules for $\beta$-carotene encapsulation
}

\author{
Rui M. Rodrigues ${ }^{\mathrm{a}}$, Philippe E. Ramos ${ }^{\mathrm{a}}$, M.F. Cerqueira ${ }^{\mathrm{b}, \mathrm{c}}$, José A. Teixeira ${ }^{\mathrm{a}}$, António A. Vicente ${ }^{\mathrm{a}}$, \\ Lorenzo M. Pastrana ${ }^{\mathrm{b}}$, Ricardo N. Pereira ${ }^{\mathrm{a}}$, Miguel A. Cerqueira ${ }^{\mathrm{b}, *}$ \\ ${ }^{a}$ CEB - Centre of Biological Engineering, University of Minho, Campus de Gualtar, 4710-057 Braga, Portugal \\ ${ }^{\mathrm{b}}$ INL - International Iberian Nanotechnology Laboratory, Av. Mestre José Veiga s/n, 4715-330 Braga, Portugal

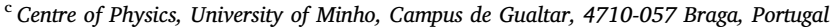

\section{A R T I C L E I N F O}

\section{Keywords:}

Nanotechnology

Encapsulation

Electrohydrodynamic process

Functional compounds

\begin{abstract}
A B S T R A C T
In this work an electrohydrodynamic process (electrospray) was used to produce $\beta$-carotene loaded nanocapsules based on whey protein isolate (WPI). WPI solutions were prepared in aqueous solutions with different concentrations of ethanol (5, 10 and 15\%) which were used for $\beta$-carotene solubilization. Different electrospray conditions were tested and the morphology and molecular organization of the nanocapsules were studied on dried and hydrated state. The size of the dried nanocapsules ranged between 227 and $283 \mathrm{~nm}$. After hydration, there was a significant increase in the mean size of the nanocapsules, being the sizes higher for nanocapsules produced with increasing concentrations of ethanol. Results, obtained from the reactivity of free sulfhydryl groups and fluorescence analysis, showed that the increase of ethanol concentration had a destabilizing effect on the protein unfolding.

Electrosprayed WPI-based nanocapsules can be used for the encapsulation of $\beta$-carotene answering the industrial demand for novel encapsulation technologies to protect sensitive bioactive compounds.
\end{abstract}

\section{Introduction}

Nanoencapsulation offers many benefits for the development of delivery systems with enhanced properties. Delivery systems at nanoscale present better physicochemical properties - i.e. optical, thermal, absorption and stability - when compared with micro- and macroscale systems, which make their use very attractive in the processing of functional foods and novel packing systems. The main aspect that oversees this behaviour is the area-to-volume ratio once it determines the physicochemical characteristics and brings several advantages, such as a high encapsulation efficiency and stability, a tailored control release, an enhanced solubility and also the prevention of undesirable chemical reactions (Cerqueira et al., 2014; Cerqueira et al., 2017).

Electrohydrodynamic process (electrospinning and electrospray) present an interesting solution for encapsulation of bioactive molecules. This methodology is able to create capsules and fibers with different sizes (at nano- and microscale) with a large surface to volume ratio, as well as to encapsulate, coat and dry the compounds in a one-step process (Anu Bhushani \& Anandharamakrishnan, 2014; Martín, LaraVillosslada, Ruiz, \& Morales, 2015). In this production process, temperature is not required, allowing the preservation of thermally labile compounds. This is possible due to an electrically-driven force used during the electrohydrodynamic processing, which leads to the formation of an electrically charged fluid cone jet (Taylor cone) of the biopolymer solution between the spinneret and the collector. This cone is obtained when the equilibrium of the electric forces and surface tension is destroyed.

The use electrohydrodynamic processes for the encapsulation of bioactive compounds have been punctually addressed. AceitunoMedina, Mendoza, Lagaron, and López-Rubio (2014) tested the encapsulation of folic acid in amaranth protein isolate (API) and pullulan electrospun fibers, reporting an increase of the thermal stability of the structure and showing that is possible to avoid folic acid degradation, assessed by the photoprotection during $120 \mathrm{~min}$. Using whey protein concentrate (WPC), López-Rubio and Lagaron (2012) showed the possibility of using the electrospray process to encapsulate $\beta$-carotene in nanoparticles, with a high encapsulation efficiency and, at the same time, allows the stabilization of $\beta$-carotene against photo-oxidation. Both lipophilic and hydrophilic bioactive compounds can be encapsulated by electrospray, but when using proteins the solubility of the lipophilic compounds can be challenging. The use of organic solvents

\footnotetext{
* Corresponding author.

E-mail address: miguel.cerqueira@inl.int (M.A. Cerqueira).
} 
can help the solubilisation and the electrospray process, however their influence on the capsule/fibre formation needs to be addressed as they can also influence the unfolding and stability of the proteins (Uzun, Kim, Leal, \& Padua, 2016). It is then important to evaluate the possibility of using ethanol as solvent, which can allow to increase the amount of the lipophilic compound encapsulated, and assess how the presence of ethanol will influence the structure of the biopolymer and capsules made thereof.

In this work, electrospray was used to produce nanocapsules based on WPI. Solutions with different concentrations of ethanol were used as solvent aiming a better solubilisation of $\beta$-carotene and a better efficiency of the electrospray process. The obtained nanocapsules were evaluated on dried and hydrated state regarding their morphology and molecular organization.

\section{Materials and methods}

\subsection{Materials}

WPI (Volactive Ultrawhey 90, Volac International Ltd, Royston, UK) was kindly donated by Nutripar, Lda (Portugal) and used without purification. WPI had the following composition: $91 \%$ of protein, $5.5 \%$ of moisture content, $3 \%$ of lactose and $0.5 \%$ of fat. 8-anilino-1-naphthalenesulfonic acid (ANS), Ellman's DTNB (5.5'-dithiobis-(2-nitrobenzoicacid), $\beta$-carotene (95\%) and ethanol (99.8\%) were obtained from Sigma-Aldrich (USA).

\subsection{Preparation of solutions}

WPI was solubilized in distilled water and mixed with ethanol to obtain three solutions with $18 \%(\mathrm{w} / \mathrm{w})$ of WPI and 5, 10 and 15\% (v/v) of ethanol. The concentration of $18 \%(\mathrm{w} / \mathrm{w})$ was selected based on previous optimization studies, as this was the concentration that allows the formation of capsules through electrospray. WPI denaturation and precipitation occurred for ethanol concentrations higher than $15 \%$ turning impossible to process the solution by electrospray.

Separately, $\beta$-carotene solutions were prepared by dissolving $\beta$ carotene in ethanol $(0.02 \mathrm{mg} / \mathrm{mL})$ and mixing with the WPI solution, through a similar procedure as described for the WPI/ethanol solutions. Three solutions with $18 \%(\mathrm{w} / \mathrm{w})$ of WPI were obtained, each with 5,10 and $15 \%(\mathrm{v} / \mathrm{v})$ of ethanol, and $0.001 \mathrm{mg} / \mathrm{mL}, 0.002 \mathrm{mg} / \mathrm{mL}$ and $0.003 \mathrm{mg} / \mathrm{mL}$ of $\beta$-carotene, respectively.

The obtained solutions were stirred overnight at room temperature, followed by centrifugation at $1774 \mathrm{~g}$ during $5 \mathrm{~min}$, to remove denatured and none-dissolved material (the obtained pellet always $<10 \%$ of the total mass used initially). Six solutions were obtained, with different concentrations of ethanol and $\beta$-carotene being designated as WPI_5, WPI_10 and WPI_15 for WPI solutions in 5, 10 and $15 \%(\mathrm{v} / \mathrm{v})$ of ethanol, and WPI_5_BC, WPI_10_BC and WPI_15_BC, for WPI for solutions in 5,10 and $15 \%(\mathrm{v} / \mathrm{v})$ of ethanol with $\beta$-carotene, respectively.

\subsection{Production of $\beta$-carotene nanocapsules and characterization}

\subsubsection{Electrospray}

Electrospray equipment (ESR200R2D, NanoNC, Korea) worked with an output voltage of $20 \mathrm{kV}$ and with a flow rate of $0.5 \mathrm{~mL} / \mathrm{h}$, using a pump were the syringe was placed vertically (EP100, NanoNC, Korea). The WPI solutions previously described were pumped through a needle with an inner diameter of $1.422 \mathrm{~mm}$, being the distance between the needle and the deposition surface of $12 \mathrm{~cm}$. These conditions were chosen based on preliminary tests where the WPI concentration, water:ethanol ratio, voltage and flow rate were varied to obtain a stable jet mode and the formation of nanocapsules. The experimental setup was held in the equipment safety cabinet at room temperature. For the collection of the capsules the collector was covered with aluminium sheet. The final dried samples were stored in the dark and at room temperature conditions until further use.

\subsubsection{Scanning electron microscopy (SEM) and Raman measurements}

The methodologies used for the evaluation of dried nanocapsules by scanning electron microscopy and Raman spectroscopy measurements are presented in Supplementary information.

\subsection{Rehydration of nanocapsules and characterization}

The electrosprayed nanocapsules were suspended in distilled water at a concentration of $0.5 \mathrm{mg} / \mathrm{mL}$. These suspensions were placed in an orbital shaker (KL-2 Edmund Buhler, Germany) at $90 \mathrm{rpm}$ for $2 \mathrm{~h}$ at room temperature to ensure full rehydration and further analysed.

2.4.1. Transmission electron microscopy (TEM), dynamic light scattering, fluorescence and reactivity of the free sulfhydryl groups

The methodologies used for the evaluation of hydrated nanocapsules by transmission electron microscopy, dynamic light scattering, fluorescence and reactivity of the free sulfhydryl groups are presented in Supplementary information.

\subsection{Statistical analysis}

The statistical analyses of the experimental data was performed through Statistica package software version 10 (StatSoft Inc., Tulsa, OK, USA). All experiments were run at least in triplicate. Statistical significance was determined by an analysis of variance (ANOVA) to at a confidence level of $95 \%$.

\section{Results and discussion}

\subsection{Size and morphology of the dried nanocapsules}

Through the visual analysis and correspondent image-based size distribution presented in Fig. 1 it was possible to verify that the electrospayed capsules presented a spherical and smooth morphology with a very broad size distribution with an average capsule size of $269 \mathrm{~nm}$. These results are in agreement with the expectations for this processing methodology and corroborate other works reported in literature. Other works using WPC resulted in capsules with sizes bellow $2 \mu \mathrm{m}$ (LópezRubio \& Lagaron, 2012; Pérez-Masiá et al., 2015). Sullivan, Tang, Kennedy, Talwar, and Khan (2014) also used ethanol (20\%, v/v) to improve the electrohydrodynamic processing of WPI, however on that study the authors aimed to obtain fibers. Nevertheless, they were able to obtain nanocapsules mentioning that at those conditions no sufficient molecular entanglement was developed to the fiber formation.

The electrosprayed capsules produced in this study presented a more regular shape and lower size distribution than the ones produced by other techniques, such as nanospray-drying (Li, Anton, Arpagaus, Belleteix, \& Vandamme, 2010). The capsules presented a size range that guarantees a high surface to volume ratio and ensures a low impact on texture and appearance upon their incorporation in foods. Moreover, produced capsules also showed a good degree of dispersion among them, suggesting low agglomeration and a free flow behaviour. The changes in ethanol concentration and $\beta$-carotene inclusion did not modify the overall morphology of the capsules, but with the increasing of ethanol concentration, there was a tendency to form larger capsules. López-Rubio and Lagaron (2012) used WPC (40\%, w/w) with different concentrations of glycerol to produce capsules by electrospray. They used glycerol to improve the solubilisation of $\beta$-carotene, and showed that the presence of glycerol led to greater median values and to a higher proportion of capsules with higher size.

\subsection{Spectroscopic analysis of the dried nanocapsules}

The Raman spectra of electrosprayed nanocapsules are presented in 


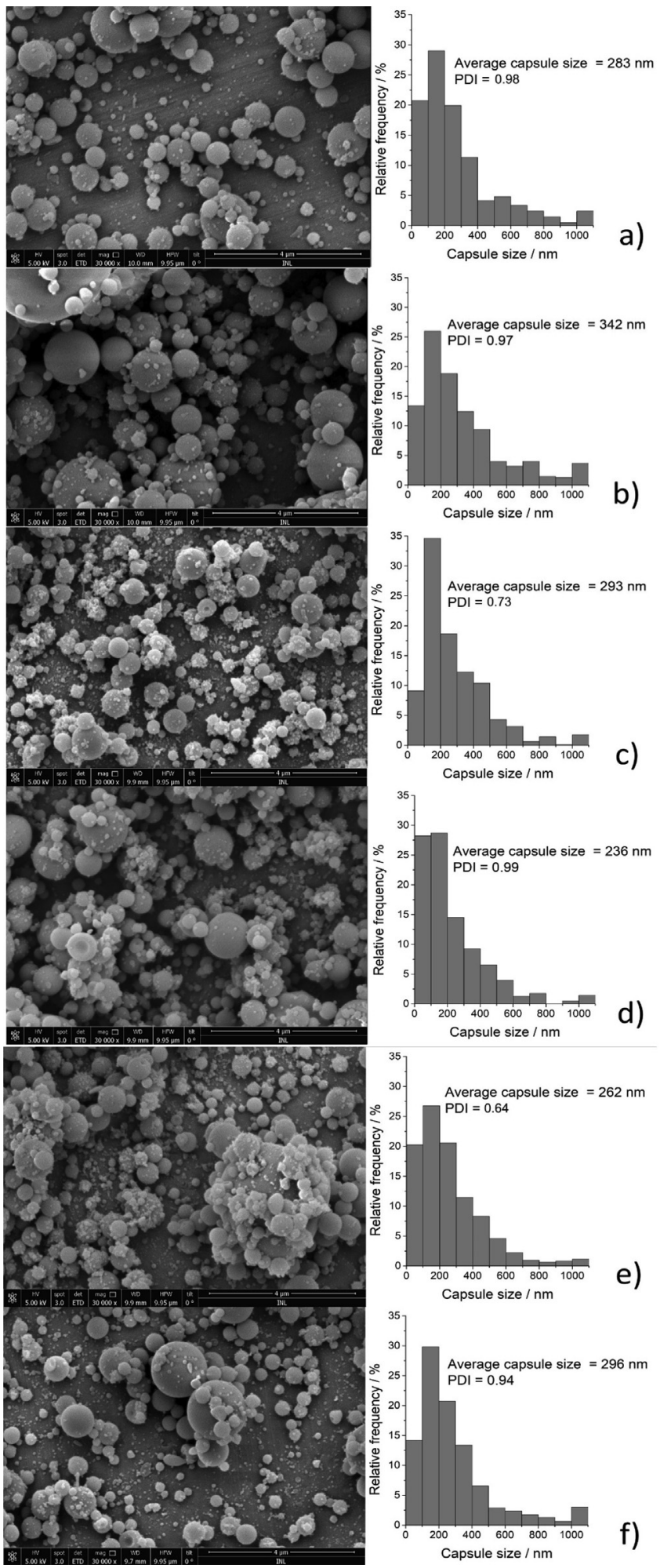

Fig. 1. SEM images of electrosprayed nanocapsules for a) WPI_5, b) WPI_10, c) WPI_15, d) WPI_5_BC, e) WPI_10_BC, f) WPI_15_BC.

Fig. 2A). Fig. 2B) shows the Raman spectra of $\beta$-carotene, WPI and one of the Raman spectra presented in Fig. 2A) for comparison. It is seen that the Raman spectra of the studied nanocapsules present the typical signatures of whey proteins. These signature lie in two ranges, namely: $900-1700 \mathrm{~cm}^{-1}$ and $3000 \mathrm{~cm}^{-1}$. The last range corresponds to the $\mathrm{CH}$ stretching vibrations of aromatic and aliphatic structures (SzymańskaChargot, Cybulska, \& Zdunek, 2011) being possible to observe that the samples do not differ in this range. The first range is quite broader and contains important structural information. The most intense peaks appear i) between 1550 and $1750 \mathrm{~cm}^{-1}$ and corresponds to amide I range and ii) in the range $1200-1350 \mathrm{~cm}^{-1}$ and corresponds to amide III region. It is well known (Jung, Czarnik-Matusewicz, \& Ozaki, 2000) that the existence of a secondary protein structure can be analysed in the amide I and amide III regions, since the frequency dependence of these bands depends strongly on the protein state, environment and the intermolecular interactions. The main intense peaks in the amide I and II regions are as follows: peak 1 at $1669 \mathrm{~cm}^{-1}$ corresponding to the $v$ $(\mathrm{C}=\mathrm{O})$ from amide $\mathrm{I}$ and $\mathrm{v}(\mathrm{C}=\mathrm{C})$; peak 2 at $\approx 1455 \mathrm{~cm}^{-1}$ that corresponds to $\delta\left(\mathrm{CH}_{2}\right)$; peak 3 at $\approx 1327 \mathrm{~cm}^{-1}$ corresponding to amide III ( $\alpha$-helix structure), $v(\mathrm{C}-\mathrm{O})$ and $\delta(\mathrm{C}-\mathrm{H})$; and the peak 4 at $\approx 1245 \mathrm{~cm}^{-1}$ that was assigned to $\beta$-sheet structure of the protein (Blanpain-Avet et al., 2012; Stephani et al., 2017). In addition, it is also seen the peak 5 at $\approx 1008 \mathrm{~cm}^{-1}$ which is attributed to $\mathrm{C}-\mathrm{CH}_{3}$ bonds. The intensity of this mode is stronger for samples WPI_5 and WPI_5BC, than for the others. This can be related to the low ethanol concentration that imply less structural change within protein structure, contrary to the cases where higher ethanol concentrations were used. $\mathrm{C}-\mathrm{CH}_{3}$ bonds are nonpolar groups present in sidechains of some amino acids, being responsible for hydrophobic interactions. For higher ethanol concentrations, the protein structure was destabilized and hydrophobic interactions were promoted, probably hindering the access to these groups.

The band shape of the amide I mode (at $\approx 1669 \mathrm{~cm}^{-1}$ ) can be considered as overlapping components corresponding to $\alpha$-helices, $\beta$ sheets, turns and random structures (Blanpain-Avet et al., 2012). Indeed the peak is asymmetric and the perfect fit of this band is achieved as a contribution of two lorentzians centred at $1669 \mathrm{~cm}^{-1}$ and $1617 \mathrm{~cm}^{-1}$. The intensity ratio between these two contributions $\left(\mathrm{I}_{1669} /\right.$ $\mathrm{I}_{1617}$ ) increases from 5.5 (for WPI_5) to 7 (WPI_15), passing by 6.5 (WPI_10). The same behaviour was observed for the BC samples, for which this ratio increases from 5.6 (WPI_5_BC) to 6.8 (WPI_10_BC) and 7.5 (WPI_15_BC).

Fig. 2C) presents the intensity ratios between the peak at $1669 \mathrm{~cm}^{-1}$ and the peaks at $1327 \mathrm{~cm}^{-1}\left(\mathrm{I}_{1669} / \mathrm{I}_{1327}=\mathrm{I}_{\mathrm{a}}\right), 1455 \mathrm{~cm}^{-1}$ $\left(\mathrm{I}_{1669} / \mathrm{I}_{1455}=\mathrm{I}_{\mathrm{b}}\right)$ and $1245 \mathrm{~cm}^{-1}\left(\mathrm{I}_{1669} / \mathrm{I}_{1245}=\mathrm{I}_{\mathrm{c}}\right)$. From the behaviour of the ratio $\mathrm{I}_{\mathrm{a}}$, related with the mode at $\approx 1327 \mathrm{~cm}^{-1}$, which is assigned to the $\mathrm{C}-\mathrm{H}$ bending or $\mathrm{CH}_{3}$ symmetric vibrations and/or to the Trp vibration (Rygula et al., 2013) it is clear the influence of ethanol in the structure of the nanocapsules. The intensity ratio $\mathrm{I}_{\mathrm{a}}$ increases with increasing ethanol concentration added to WPI solution. In this case the increase of intensity can be related with the high exposure of protein hydrophobic groups.

During the capsule formation process the proteins were exposed to ethanol resulting in their structural destabilization and increased unfolding. However, the almost constant intensity ratio $\mathrm{I}_{\mathrm{b}}$ (between $1699 \mathrm{~cm}^{-1}$ and $1455 \mathrm{~cm}^{-1}$ modes) - being the peak $1455 \mathrm{~cm}^{-1}$ assigned to the $\mathrm{C}=\mathrm{O}$ group of Asp and Glu carboxyl groups (Rygula et al., 2013) - do not indicates significant changes in the main protein structure. The intensity of the band at $\approx 940 \mathrm{~cm}^{-1}$ is proportional to the $\alpha$-helix content (Ikeda \& Li-Chan, 2004; Ikeda, 2003). The intensity of this band in the measured Raman spectra is very low, so it can be concluded that the $\alpha$-helix content is low and do not significantly changes from sample to sample. Furthermore, it is also known (Chi, Chen, Holtz, \& Asher, 1998) that the intensity of a band at $\approx 1395 \mathrm{~cm}^{-1}$ decrease as the $\alpha$-helix content increases, but unfortunately it was not possible to observe this band. Moreover, the band at $\approx 1245 \mathrm{~cm}^{-1}$ assigned to $\beta$-sheet structures is clearly seen in the Raman spectra and the intensity ratio $\mathrm{I}_{\mathrm{c}}\left(=\mathrm{I}_{1669} / \mathrm{I}_{1245}\right)$, shows a peculiar behaviour, as shown in Fig. 2c). Interestingly, the intensity ratio between $1327 \mathrm{~cm}^{-1}$ band and $1245 \mathrm{~cm}^{-1}$ band (not shown here) follows the same behaviour, i.e., this ratio increases with the increase of the ethanol content for the $\mathrm{BC}$ samples, but in the samples without $\beta$ carotene, it is seen an increase between 5 and 10\% followed by a decrease for $15 \%$. Eventually this change can be related with any transient 


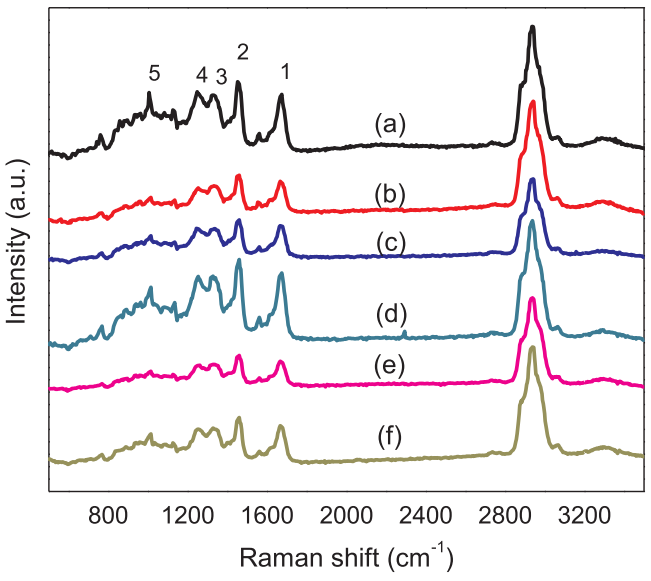

(A)

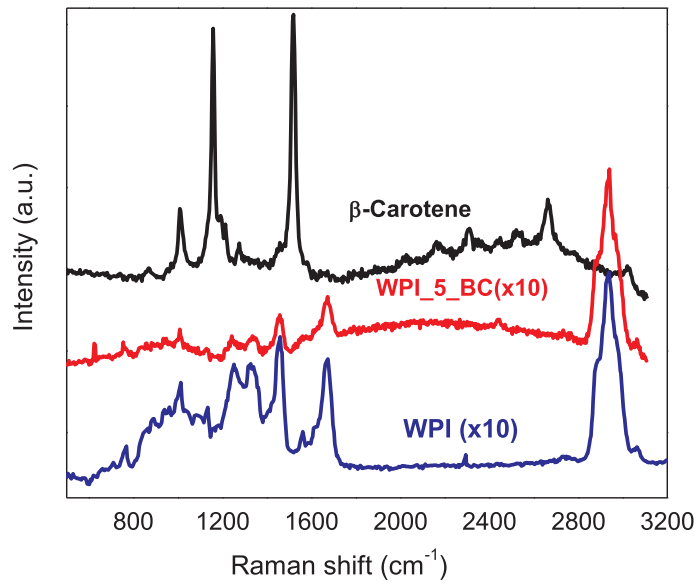

(B)

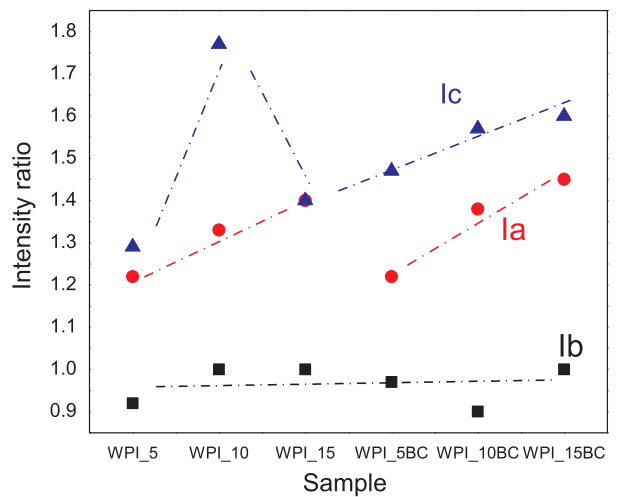

(C)

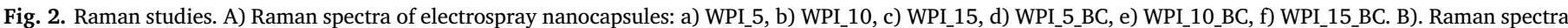

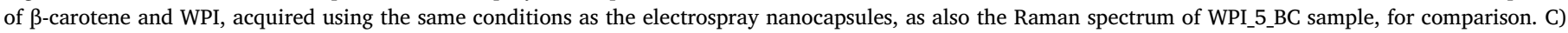

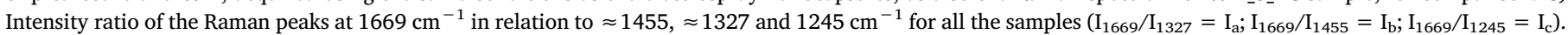
The lines are guides to the eye.

structural conformation adopted by the protein but better elucidation through other study is needed to confirm this hypothesis.

\subsection{Size and morphology of the hydrated nanocapsules}

The stability of the capsules is an important factor for their successful incorporation in food matrices for further consumption. TEM imaging was used in order to evaluate stability of capsules after rehydration. The TEM images presented in Fig. 3 clearly show the presence of the capsules, the maintenance of their spherical morphology after rehydration, and confirm that the capsule size is in the range observed by SEM i.e.: WPI_5 - $180 \pm 30 \mathrm{~nm}$, WPI_10 $-160 \pm 60 \mathrm{~nm}$, WPI_15 $290 \pm 100 \mathrm{~nm}$, WPI_5_BC - $240 \pm 60 \mathrm{~nm}$, WPI_10_BC $130 \pm 30 \mathrm{~nm}$ and WPI_15_BC $-140 \pm 40 \mathrm{~nm}$ ).

Once verified the stability and morphological features of the rehydrated capsules, DLS analysis was performed. Table 1 presents the values of Z-average, PdI and $\zeta$-potential of the capsules suspended in water. The results obtained by DLS are different from the ones obtained by TEM and SEM (both TEM and SEM are performed on dried capsules) showing high sizes for the capsules suspended in water. Results obtained by DLS showed that for capsules suspended in water occurs an increase of the size and polydispersity for samples with higher concentrations of ethanol. Ethanol acts as a co-solvent, changing the equilibrium between the polar and non-polar groups within the protein, destabilizing proteins folding's (Nick Pace, Trevino, Prabhakaran, \& Martin Scholtz, 2004). For higher concentrations of ethanol, the proteins in the solution presented a higher degree of unfolding, resulting on a higher exposure of its reactive groups and thus potentiating aggregation.

DLS results also showed that the incorporation of $\beta$-carotene resulted in the increase of the size of the capsules when they are suspended in water. The polydispersity of the capsules containing $\beta$-carotene is also higher for the 5 and $10 \%$ ethanol concentrations and similar at $15 \%$. The increase in capsule size with the incorporation of $\beta$ carotene was reported previously with electrosprayed $\beta$-carotene loaded in whey protein concentrate systems (López-Rubio \& Lagaron, 2012). In this study the increase in capsule size was only noticed by DLS measurements, reflecting changes between 200 and $300 \mathrm{~nm}$ in the capsule average size. The fact that this size differences were not observed on SEM and TEM images (both SEM and TEM are performed on dried capsules) can be explained by the different swelling behaviour on the capsules containing $\beta$-carotene. Being $\beta$-carotene a hydrophobic compound, it is expected to interact with the hydrophobic groups on the proteins. By interacting with these groups, hydrophobic proteinprotein interactions - that play an essential role on protein aggregation - may be affected. In this case, the $\beta$-carotene is likely to impact the aggregation mechanism and the general properties of the capsules as is the case of the swelling capacity.

\subsection{Free SH groups}

Protein unfolding results on the exposure of reactive groups, allowing them to react and contribute to the aggregation process. The disulphide bridges are the strongest bonds involved on the protein 

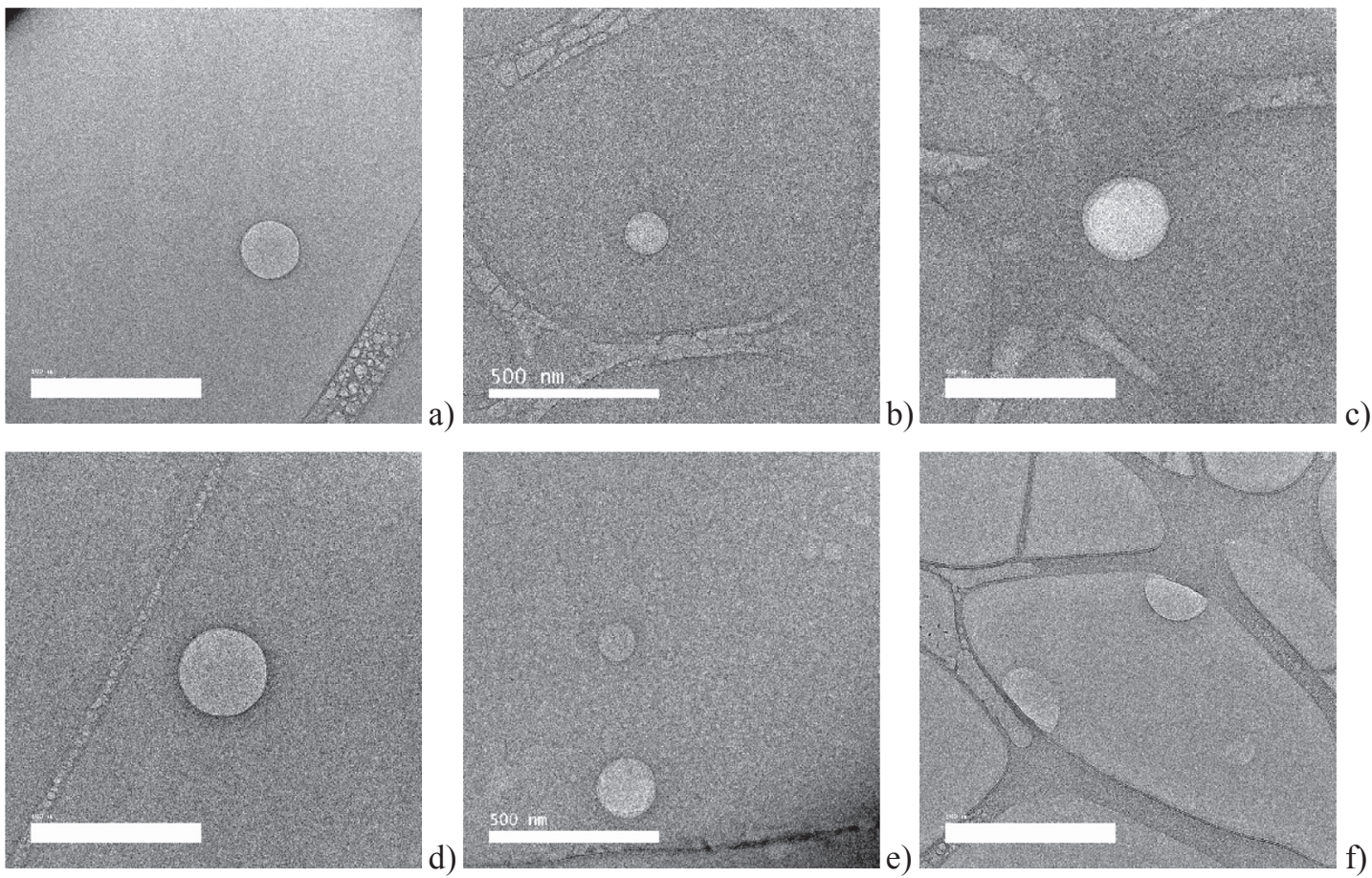

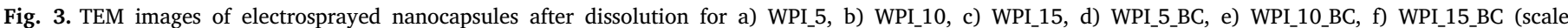
bar $=500 \mathrm{~nm})$.

Table 1

Z-Average, polydispersity (PdI) and $\zeta$-potential of the capsules suspended in water.

\begin{tabular}{llll}
\hline Sample & Z-Average $(\mathrm{nm})$ & PdI & \multicolumn{1}{l}{ S-potential } \\
\hline WPI_5 & $414.7 \pm 34.04^{\mathrm{a}}$ & $0.445 \pm 0.006^{\mathrm{a}}$ & $-25.5 \pm 2.3^{\mathrm{a}}$ \\
WPI_10 & $536.23 \pm 54.36^{\mathrm{b}}$ & $0.573 \pm 0.071^{\mathrm{b}}$ & $-27.1 \pm 1.8^{\mathrm{a}}$ \\
WPI_15 & $865.05 \pm 36.10^{\mathrm{c}}$ & $0.658 \pm 0.045^{\mathrm{bc}}$ & $-25.4 \pm 0.4^{\mathrm{a}}$ \\
WPI_5_BC & $643.65 \pm 30.33^{\mathrm{d}}$ & $0.568 \pm 0.023^{\mathrm{b}}$ & $-24.6 \pm 2.0^{\mathrm{a}}$ \\
WPI_10_BC & $863.45 \pm 76.29^{\mathrm{c}}$ & $0.668 \pm 0.021^{\mathrm{c}}$ & $-24.3 \pm 1.7^{\mathrm{a}}$ \\
WPI_15_BC & $1016.78 \pm 20.64^{\mathrm{d}}$ & $0.661 \pm 0.033^{\mathrm{c}}$ & $-26.9 \pm 1.9^{\mathrm{a}}$ \\
\hline
\end{tabular}

For each column, different letters correspond to statistical significance differences $(p<0.05)$.

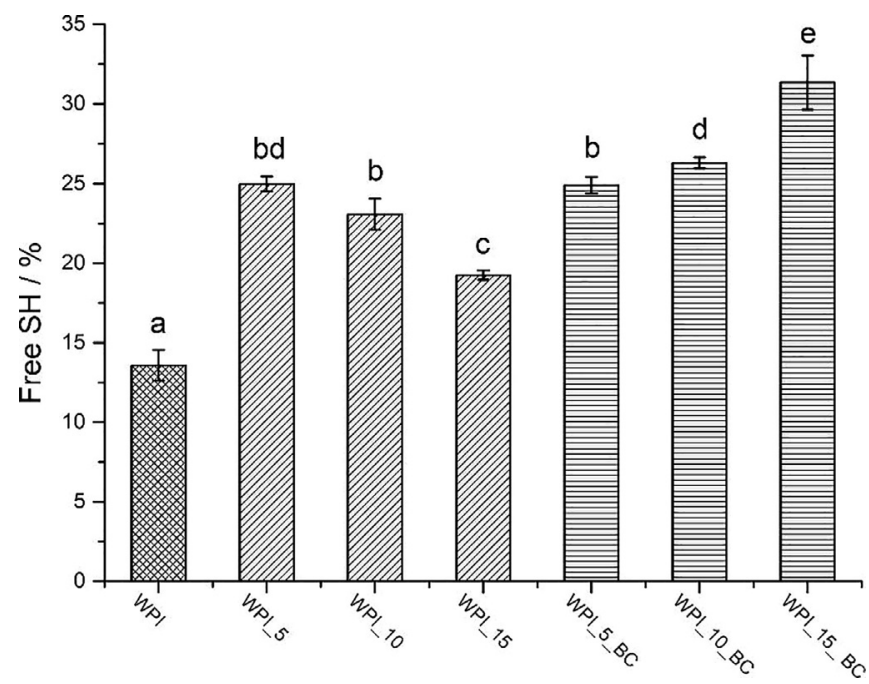

Fig. 4. Percentage of free SH groups in WPI solution and produced capsules. For each column, different letters correspond to statistical significance differences $(p<0.05)$. aggregation process, actively contributing to the structural and functional properties of protein based structures. By determining the accessibility of free $\mathrm{SH}$ groups is possible to estimate the extent of protein unfolding and $\mathrm{SH}$ crosslinking. Fig. 4 presents the percentage of free $\mathrm{SH}$ groups in the WPI solution and capsules produced by electrospray after hydration.

In preliminary tests (results not shown) capsules produced without the presence of ethanol presented a decrease of the free $\mathrm{SH}$ groups when compared with the control solution. On the other hand, the WPI-based capsules suspension produced with the presence of ethanol present a higher amount of free $\mathrm{SH}$, that can result from a higher degree of unfolding. For WPI-based capsules the content of free SH decreased for higher concentrations of ethanol. This behaviour can be related with the increasing unfolding and consequent exposure of $\mathrm{SH}$; these groups are more likely to react among them giving rise to disulphide bridges, reducing then the free $\mathrm{SH}$. On the capsules containing $\beta$-carotene it was observed the opposite behaviour; for higher concentrations of ethanol and $\beta$-carotene the free $\mathrm{SH}$ also increases. This can be explained by the interaction between $\beta$-carotene and protein hydrophobic regions which affected the interaction and aggregation of the proteins, thus impairing the disulphide crosslinking. This is in agreement with the behaviour of the $\beta$-carotene loaded capsules that showed higher PDI values (DLS results); this can be justified by the lower degree of disulphide crosslinking which results on "weaker" and less rigid capsule matrices, being a possible cause for a higher swelling on the capsules containing $\beta$ carotene, and thus contributing to less standardization of capsules size.

\subsection{Fluorescence determinations}

Fluorescence spectroscopy is a simple and efficient way to characterize molecular microenvironments and local structural transitions. Intrinsic fluorescence of proteins, results from fluorescent aminoacid residues as tryptophan, being used to probe tertiary structural changes. During the spectra analysis (see Fig. 5 insert), the maximum emission intensity was at $330 \mathrm{~nm}$ for all samples tested, suggesting that tryptophan microenvironment was a polar and no changes on the polarity took place. On other hand, the fluorescence intensity, suffered 


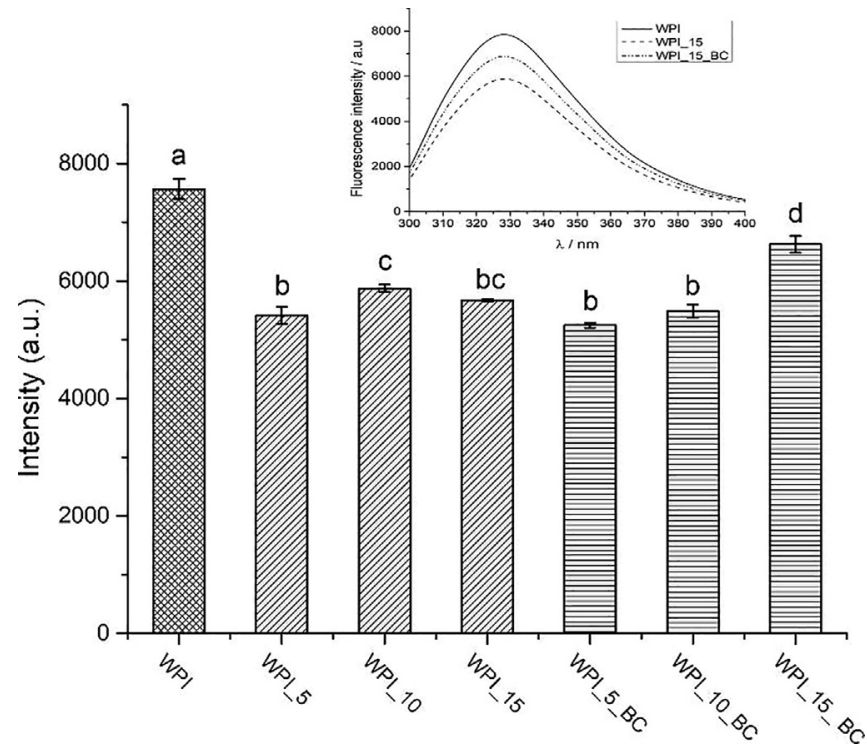

Fig. 5. Intrinsic fluorescence in WPI solution and produced capsules. For each column, different letters (a-d) correspond to statistical significance differences $(p<0.05)$. Figure insert shows representative spectra (WPI, WPI_15 and WPI_15_BC).

significant changes with the process of capsule formation, as is presented in Fig. 5. This changes in fluorescence intensity reflect changes in fluorescent groups exposure and quenching; either from selfquenching (i.e. neighbour aminoacids and disulphide bonds) or quenching by the solvent and other molecules present on the medium (Lakowicz, 2006).

It is observed a clear differentiation between the control WPI solution and the electrosprayed capsules suspension. The decrease of the fluorescence of the capsule suspensions when compared with the control solution may reflect the protein unfolding and higher exposure of the fluorescent groups to the medium, thus being susceptible to be quenched by the solvent and medium molecules (Lakowicz, 2006). The opposite effect may be caused by the capsule size increase once the proteins inside the capsules are shielded from the medium. In this sense, the capsules fluorescence may reflect the increase of ethanol concentration and capsule size variations. For samples without $\beta$-carotene this increase is significant $(p<0.05)$ for the capsules produced with $10 \%$ ethanol when compared to the capsules produced with $5 \%$ ethanol, while the higher content of disulphide bonds may have contributed to reduce the fluorescence in samples produced at $15 \%$ ethanol. Particularly the system with a notorious higher fluorescence is the one produced with $15 \%$ ethanol with $\beta$-carotene. This system presented not only higher capsules size, but also was the one with markedly higher amount of free SH groups. As previously stated, this implies lower concentration of disulphide bonds, known to be strong quenchers (Stănciuc, Aprodu, Râpeanu, \& Bahrim, 2012), thus contributing to an increase of the fluorescence intensity.

The use of the ANS (8-anilino-1-naphthalenesulfonic acid) as extrinsic fluorescent probe allows to characterize the accessibility of the hydrophobic sites. ANS is slightly fluorescent on its free form but it has a great affinity with the hydrophobic sites of the proteins and when bound to them it markedly increases its fluorescence. ANS-protein fluorescence, presented in Fig. 6, was used to characterize the WPI control solution and the capsule's suspensions.

During the capsule formation process the proteins are exposed to ethanol resulting in their structural destabilization and increased unfolding. As mentioned before, this unfolding also results on the exposure of hydrophobic clusters, which are essential to the aggregation and capsule production. This is also responsible by the higher fluorescence of ANS in the capsules systems, compared to the control WPI

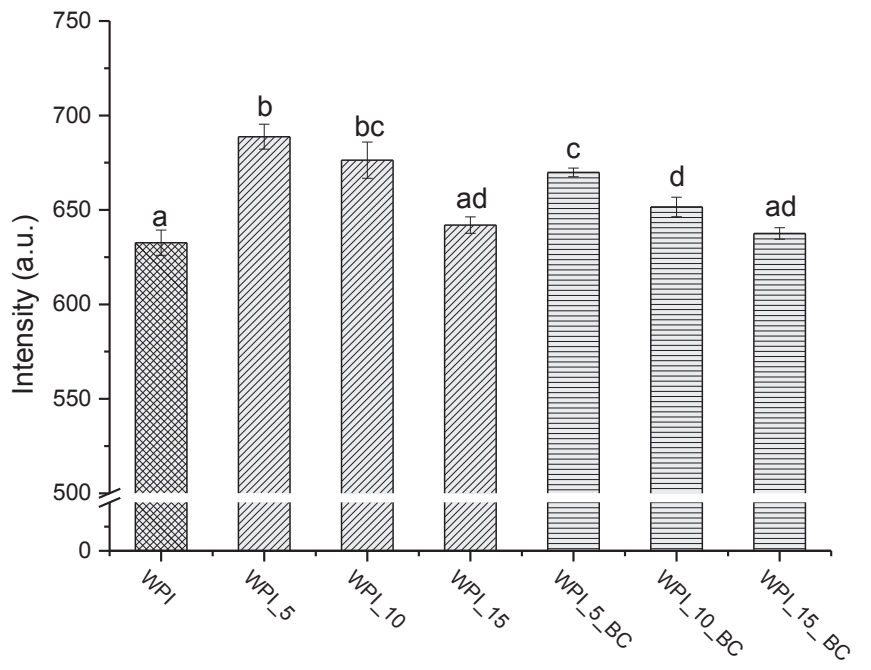

Fig. 6. ANS fluorescence in WPI solution and produced capsules. For each column, different letters (a-d) correspond to statistical significance differences $(p<0.05)$.

solution, since hydrophobic groups are more accessible after ethanol exposure. The increasing of ethanol concentration resulted on higher unfolding and more exposure of hydrophobic clusters, making them more likely to interact and be occupied as part of the capsules structure, resulting on the decrease of ANS fluorescence. The samples containing $\beta$-carotene presented the same tendency of the ones produced only with ethanol, being the ANS fluorescence reduced with the increasing of ethanol concentration. Despites that the fluorescence intensity can be significantly lower for the samples containing $\beta$-carotene at 5 and $10 \%$ of ethanol, these outcomes depend on the ethanol concentration used. This shows that the hydrophobic nature of $\beta$-carotene may play an important role on protein structural rearrangements, blocking the hydrophobic sites on the capsulés structure and competing with ANS binding.

\section{Conclusion}

WPI-based nanocapsules can be obtained by electrospray and used for the encapsulation of $\beta$-carotene. The use of aqueous solutions with increasing concentrations of ethanol improve the process throughput, and helps the solubilisation of lipophilic compounds, such as $\beta$-carotene The capsules produced presented spherical shape and sizes in nano and microscale range. These capsules are stable once suspended in water, retaining their shape and size range. The presence of ethanol and $\beta$ carotene influences protein structural conformation, affecting the aggregation and capsule formation process. Ethanol contributes for the destabilization of the protein folding allowing to $\beta$-carotene to interact with the proteińs hydrophobic core. All along, these factors impact capsule size as well as intramolecular interactions between the proteins.

\section{Author contributions section}

R.M. Rodrigues, P.E. Ramos, M.F. Cerqueira, R.N. Pereira and M.A. Cerqueira have contributed to the acquisition, analysis and interpretation of the data as well as for the drafting and revision of the manuscript. J.A. Teixeira, A.A. Vicente and L.M. Pastrana have revised the manuscript and contributed to the discussion of the results. All the authors revised and approved the final version of the manuscript.

\section{Declaration of Competing Interest}

The authors declare that they have no known competing financial interests or personal relationships that could have appeared to 
influence the work reported in this paper.

\section{Acknowledgments}

This work was supported by the Portuguese Foundation for Science and Technology (FCT) under the scope of the strategic funding of UIDB/ 04469/2020 unit and by BioTecNorte operation (NORTE-01-0145FEDER-000004) funded by the European Regional Development Fund under the scope of Norte2020 - Programa Operacional Regional do Norte. The author Rui M. Rodrigues thanks to FCT the financial grant with SFRH/BD/110723/2015. This work was supported by the project ARMAdilhas seletivas para eliminação da VESPA velutina. Medida 6 Investigação e Desenvolvimento $\left(\mathrm{n}^{\circ}\right.$ 5894057). Instituto de Financiamento de Agricultura e Pescas, I.P.

\section{Appendix A. Supplementary data}

Supplementary data to this article can be found online at https:// doi.org/10.1016/j.foodchem.2019.126157.

\section{References}

Aceituno-Medina, M., Mendoza, S., Lagaron, J. M., \& López-Rubio, A. (2014) Photoprotection of folic acid upon encapsulation in food-grade amaranth (Amaranthus hypochondriacus L.) protein isolate - Pullulan electrospun fibers. LWT Food Science and Technology, 62, 970-975. https://doi.org/10.1016/j.lwt.2015.02. 025.

Anu Bhushani, J., \& Anandharamakrishnan, C. (2014). Electrospinning and electrospraying techniques: Potential food based applications. Trends in Food Science \& Technology, 38(1), 21-33. https://doi.org/10.1016/j.tifs.2014.03.004.

Blanpain-Avet, P., Hédoux, A., Guinet, Y., Paccou, L., Petit, J., Six, T., \& Delaplace, G. (2012). Analysis by Raman spectroscopy of the conformational structure of whey proteins constituting fouling deposits during the processing in a heat exchanger. Journal of Food Engineering, 110(1), 86-94. https://doi.org/10.1016/j.jfoodeng.2011. 12.005.

Cerqueira, M. A. Pinheiro, A. C. B., do Carmo, C. V. S., Duarte, C. M. M., da Cunha, M. das G. C., ... Vicente, A. A. M. de O. S. (2017). Nanostructured biobased systems for nutrient and bioactive compounds delivery. In Nutrient delivery (pp. 43-85). Elsevier. https://doi.org/10.1016/B978-0-12-804304-2.00002-0.

Cerqueira, M. A., Pinheiro, A. C., Silva, H. D., Ramos, P. E., Azevedo, M. A., Flores-López, M. L., ... Vicente, A. A. (2014). Design of bio-nanosystems for oral delivery of functional compounds. Food Engineering Reviews, 6(1-2), 1-19. https://doi.org/10.1007/ s12393-013-9074-3.

Chi, Z., Chen, X. G., Holtz, J. S. W., \& Asher, S. A. (1998). UV resonance raman-selective amide vibrational enhancement: quantitative methodology for determining protein secondary structure. Biochemistry, 37(9), 2854-2864. https://doi.org/10.1021/ bi971160z.
Ikeda, S. (2003). Heat-induced gelation of whey proteins observed by rheology, atomic force microscopy, and Raman scattering spectroscopy. Food Hydrocolloids, 17(4), 399-406. https://doi.org/10.1016/S0268-005X(03)00033-X.

Ikeda, S., \& Li-Chan, E. C. Y. (2004). Raman spectroscopy of heat-induced fine-stranded and particulate $\beta$-lactoglobulin gels. Food Hydrocolloids, 18(3), 489-498. https://doi org/10.1016/j.foodhyd.2003.07.003.

Jung, Y. M., Czarnik-Matusewicz, B., \& Ozaki, Y. (2000). Two-dimensional infrared, twodimensional Raman, and two-dimensional infrared and Raman heterospectral correlation studies of secondary structure of $\beta$-lactoglobulin in buffer solutions. The Journal of Physical Chemistry B, 104(32), 7812-7817. https://doi.org/10.1021/ jp0008041.

Lakowicz, J. R. (2006). Principles of Fluorescence Spectroscopy. (J. R. Lakowicz, Ed.), Principles of fluorescence spectroscopy, Springer, New York, USA, 3rd ed., 2006. Boston, MA: Springer US. https://doi.org/10.1007/978-0-387-46312-4.

Li, X., Anton, N., Arpagaus, C., Belleteix, F., \& Vandamme, T. F. (2010). Nanoparticles by spray drying using innovative new technology: The Büchi Nano Spray Dryer B-90. Journal of Controlled Release, 147(2), 304-310. https://doi.org/10.1016/j.jconrel. 2010.07.113.

López-Rubio, A., \& Lagaron, J. M. (2012). Whey protein capsules obtained through electrospraying for the encapsulation of bioactives. Innovative Food Science \& Emerging Technologies, 13, 200-206. https://doi.org/10.1016/j.ifset.2011.10.012.

Martín, M. J., Lara-Villosslada, F., Ruiz, M. A., \& Morales, M. E. (2015). Microencapsulation of bacteria: A review of different technologies and their impact on the probiotic effects. Innovative Food Science and Emerging Technologies, 27, 15-25. https://doi.org/10.1016/j.ifset.2014.09.010.

Nick Pace, C., Trevino, S., Prabhakaran, E., \& Martin Scholtz, J. (2004). Protein structure, stability and solubility in water and other solvents. Philosophical Transactions of the Royal Society B: Biological Sciences, 359(1448), 1225-1235. https://doi.org/10.1098/ rstb.2004.1500.

Pérez-Masiá, R., López-Nicolás, R., Periago, M. J., Ros, G., Lagaron, J. M., \& López-Rubio, A. (2015). Encapsulation of folic acid in food hydrocolloids through nanospray drying and electrospraying for nutraceutical applications. Food Chemistry, 168, 124-133. https://doi.org/10.1016/j.foodchem.2014.07.051.

Rygula, A., Majzner, K., Marzec, K. M., Kaczor, A., Pilarczyk, M., \& Baranska, M. (2013). Raman spectroscopy of proteins: A review. Journal of Raman Spectroscopy, 44(8), 1061-1076. https://doi.org/10.1002/jrs. 4335.

Stănciuc, N., Aprodu, I., Râpeanu, G., \& Bahrim, G. (2012). Fluorescence spectroscopy and molecular modeling investigations on the thermally induced structural changes of bovine $\beta$-lactoglobulin. Innovative Food Science \& Emerging Technologies, 15, 50-56. https://doi.org/10.1016/j.ifset.2012.03.001.

Stephani, R., Oliveira, K. de S., de Almeida, C. E. R., Perrone, Í. T., de Carvalho, A. F., de Oliveira, L. F. C., \& Almeida, M. R. (2017). Raman spectroscopy as a tool to identify modification of whey protein concentrate (WPC) during shelf life. Food Packaging and Shelf Life, 11, 1-9. https://doi.org/10.1016/j.fpsl.2016.10.001.

Sullivan, S. T., Tang, C., Kennedy, A., Talwar, S., \& Khan, S. A. (2014). Electrospinning and heat treatment of whey protein nanofibers. Food Hydrocolloids, 35, 36-50. https://doi.org/10.1016/j.foodhyd.2013.07.023.

Szymańska-Chargot, M., Cybulska, J., \& Zdunek, A. (2011). Sensing the structural differences in cellulose from apple and bacterial cell wall materials by Raman and FT-IR spectroscopy. Sensors, 11(6), 5543-5560. https://doi.org/10.3390/s110605543.

Uzun, S., Kim, H., Leal, C., \& Padua, G. W. (2016). Ethanol-induced whey protein gels as carriers for lutein droplets. Food Hydrocolloids, 61, 426-432. https://doi.org/10. 1016/j.foodhyd.2016.05.013. 